\title{
Efficacy and safety of goserelin combined with adjuvant chemotherapy in premenopausal women with breast cancer
}

\author{
Yang Wang1, Zheng Fei Zhu', Wei Jun Pan² and Zhe Xu' \\ ${ }^{1}$ Department of Pharmacy, Maanshan Municipal Health Hospital For Women And Children, Maanshan, \\ Anhui 243 000, China; ${ }^{2}$ Obstetrics, Maanshan Municipal Health Hospital For Women And Children, \\ Maanshan, Anhui 243 000, China.
}

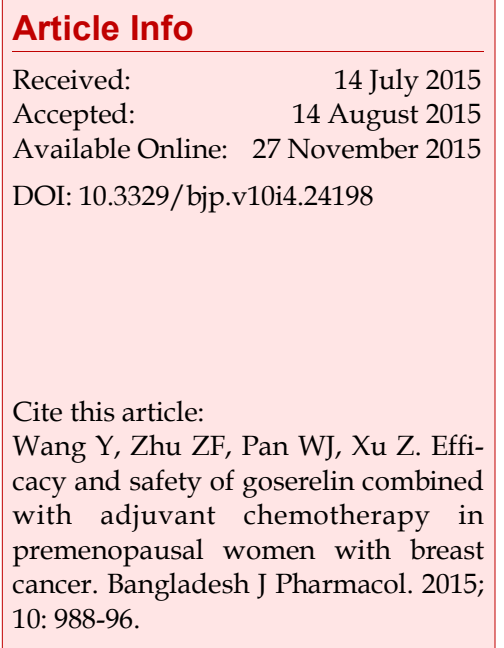

\section{Abstract}

This study aims to evaluate the efficacy and safety of goserelin combined with chemotherapy for premenopausal women with breast cancer. Literatures were extracted from databases including Excerpta Medica Database, Springer, Pubmed, China National Knowledge Infrastructure and Chinese Biological Medicine from their inception up to May 2014. The main efficacy measures were 5 years overall survival (OS), 10 years OS, 5 years disease free survival and 5 years progress free survival. Ten randomized comparison clinical trials were eligible in this study. The result showed that goserelin combined with chemotherapy group can improve the survival rate and decrease the incidence of arthralgia in postmenopausal breast cancer patients, respectively, compared to the control group. However, they can increase the occurrence of vomiting during the chemotherapy process. Compared with the simple chemotherapy, goserelin combined with chemotherapy can provide benefits for premenopausal women with breast cancer on improving the survival rate and reducing arthralgia.

\section{Introduction}

The figures released by International Agency for Research on Cancer show that breast cancer is one of the most common cancers (DeSantis et al., 2014), and the most common malignant tumor for women patients, with the rising mortality rate year by year. At present, the annual growth rate of breast cancer incidence in China is 3-4\%, higher than the average one of global growth. Meanwhile the onset age of breast cancer of Chinese women is less than that of Western women (Hong-Li et al., 2014). In clinical practice, chemotherapy is an important therapy method, but prone to cause cancer metastasis and serious adverse reactions which influence the quality of life of patients, and therefore it is necessary to find a suitable therapy method for breast cancer specific to Chinese women patients (Gielen et al., 2005).
Goserelin is a synthetic analogue of a naturally occurring luteinizing-hormone releasing hormone, which is studied most widely for treating premenopausal patients of breast cancer, and it is a very efficient kind of medicine for endocrine therapy with little toxic effect, which inhibits the secretion of luteinizing hormone (LH) and follicle-stimulating hormone (FSH) and forms a reversible inhibition of the secretion of $\mathrm{FSH}$ and $\mathrm{LH}$, to achieve the hypophysectomy effect of selective drugs and the overall suppression of ovarian function, so that the estrogen levels of premenopausal women reach the ones of postmenopausal women to inhibit the tumorgrowth-promoting effect of estrogen (Liu et al., 2013; Yang et al., 2013). Therefore, goserelin is commonly used in the adjuvant therapy of premenopausal breast cancer patients, and for the patients with estrogen receptor-positive lymph node metastasis, the same effect can be obtained as chemotherapy. 
Currently, a large number of clinical studies in goserelin have been reported. But there are obvious differences because the size of a single test sample is too small, and interventions and results index adopted in each study are inconsistent. Meta-analysis is applied in this study to comprehensively evaluate the treatment of goserelin combined with chemotherapy for premenopausal women patients, providing more reliable medical evidence for clinical application.

\section{Materials and Methods}

\section{Identification of eligible studies}

Literatures were extracted from databases including Excerpta Medica Database (EMBASE), Springer, PubMed, China National Knowledge Infrastructure (CNKI) and Chinese Biological Medicine (CBM) from their inception up to May 2014. The search terms were used as follows: 'Goserelin', 'zoladex', 'chemotherapy' and 'premenopausal women with breast cancer'.

\section{Inclusion and exclusion criteria}

Inclusion criteria include a) randomized clinical trial (RCT); b) premenopausal women with breast cancer; c) the treatment with goserelin combined chemotherapy. Exclusion criteria include a) male breast cancer patients; b) different endpoint or other types of breast diseases; c) patients had previously subjected to chemotherapy, radiotherapy or surgery; d) concurrent infection; e) other malignancy or serious medical illnesses.

\section{Data extraction}

In order to maintain uniformity and reduce potential reporting bias, two independent reviewers extracted data using a standardized collection according to the inclusion and exclusion criteria listed earlier from those databases. The following characteristics were extracted from each study: name of first author, year of publication, location in which the studies were performed, total number of cases and controls, methods of randomization, intervention and treatment duration. If there were discrepancies in all cases, they were discussed between the reviewers before a final consensus was reached. Disagreements were resolved by the third author.

\section{Statistical analysis}

Meta-analysis was performed according to the Cochrane Handbook for Systematic Reviews of Interventions. The pooled relative ratio (RR) and $95 \%$ confidence interval (CI) were used to assess efficacy and safety endpoints. Heterogeneity was analyzed using the I2 statistic: I2 $=100 \% \times(\mathrm{Q}-\mathrm{df}) / \mathrm{Q}$. I2 of $0-25 \%$ was considered to not have heterogeneity; I2 of $25-50 \%$ may represent low heterogeneity; I2 of $50-75 \%$ may represent moderate heterogeneity; and I2 of $75-100 \%$ indicate high heterogeneity (Ai et al., 2014; Lyu et al., 2014).
When no heterogeneity or low heterogeneity was present, the fixed effects model was used for meta-analysis (Chai and Liu, 2014). When moderate heterogeneity or high heterogeneity was present, the fixed effects model was compared to the random effects model of DerSimonian-Laird (Higgins and Thompson, 2002). Publication bias was assessed using funnel plots with visual inspection of asymmetry. RR, tests for heterogeneity, and forest plots for the relevant comparisons were performed using STATA 12 with Beggr's bias test and Egger's bias test, with $\mathrm{p} \leq 0.05$ indicating potential bias (Yang et al., 2013).2013).

\section{Results}

\section{Eligible studies}

1644 publications were initially identified, 1634 studies were excluded, as they were review papers contained no original data, or a reanalysis of data, or measured deferent endpoints, and/or had no control group. Finally, 10 studies (Baum et al., 2006; CastiglioneGertsch et al., 2003; Cheng et al., 2012; Davidson et al., 2005; Gerber et al., 2011; Hackshaw et al., 2009; Karlsson et al., 2011; Kaufmann et al., 2007; Kaufmann et al., 2003; von Minckwitz et al., 2006) with a total sample population of 11171 patients (5433 in the treatment group and 5738 in the control group) were identified based on the inclusion/exclusion criteria (Figure 1). Characteristics of studies included in the current metaanalysis were presented (Table I).

\section{Quality assessment}

All RCTs mentioned random allocation, 8 studies reported and described random allocation methods. None of the studies described the blind method. These RCTs design all include a baseline assessment including patients' age, stage of disease, etc. No significant differences were found between the baseline data. In these RCTs, three combination chemotherapies have been performed to treat advanced breast cancer: one study used CAF; seven studies used CMF; and the other studies used chemotherapy (Table I).

\section{Meta-analysis}

The main treatment efficacy indicators considered in meta-analysis are: a) 5 years overall survival (OS); b) 10 years OS; c) 5 years disease free survival (DFS); d) 5 years progress free survival (PFS); The indicators for the safety of the treatments are mainly the rate of occurrence of emesis and arthralgia.

\section{5 years OS of patients}

Ten clinical trials evaluated the 5 years OS of patients. Heterogeneity was considered absent at the I ${ }^{2}$ statistic $\left(\mathrm{I}^{2}=2 \%, \mathrm{p}=0.42\right)$. A fixed effects model was performed on outcome measurements. The results show that goserelin combined with chemotherapy could 


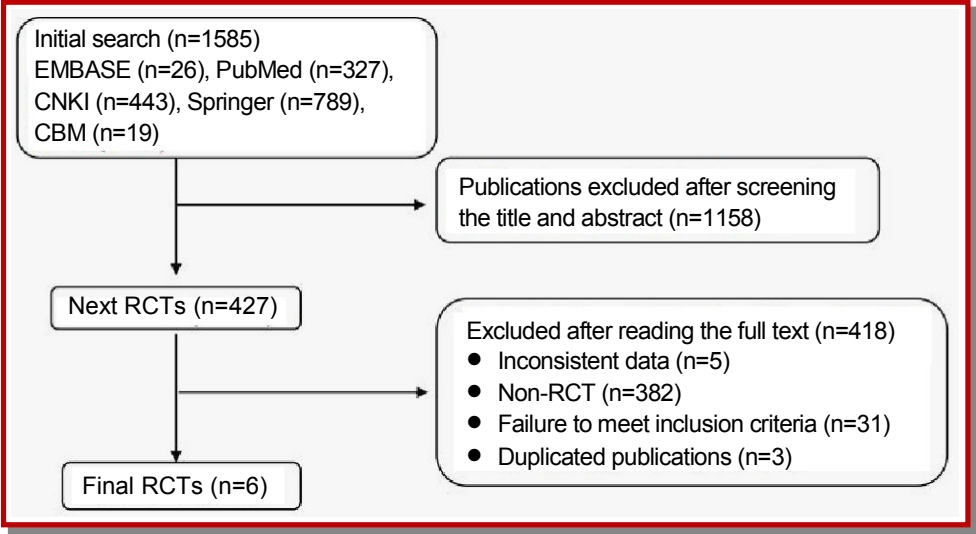

Figure 1: Flow diagram of study identification

\section{Table I}

\section{Baseline characteristics of the eligible trials}

\begin{tabular}{|l|c|c|c|c|c|c|}
\hline Study & Year & $\begin{array}{c}\text { Sample size } \\
\text { (case/control) }\end{array}$ & Age & $\begin{array}{r}\text { Intervention group } \\
\text { Test }\end{array}$ & $\begin{array}{c}\text { Treatment } \\
\text { Control }\end{array}$ & $\begin{array}{c}\text { Random alloca- } \\
\text { duration }\end{array}$ \\
\hline tion method
\end{tabular}

G: GOS; C: chemotherapy; CAF: cyclophosphamide, doxorubicin, and fluorouracil; CMF: cyclophosphamide, methotrexate, and 5-fluorouracil

significantly increase the efficacy of 5 years OS in premenopausal women with breast cancer $(\mathrm{RR}=1.07$; $95 \%$ CI $[1.05,1.09], \mathrm{p}<0.00001)$, compared to the control group (Figure 2).

\section{Ten years OS of patients}

Ten clinical trials evaluated the 10 years OS of patients. Heterogeneity was considered absent at the I2 statistic $\left(\mathrm{I}^{2}=7 \%, \mathrm{p}=0.36\right)$. A fixed effects model was performed on outcome measurements. The results show that goserelin combined with chemotherapy could significantly increase the efficacy of 10 years OS in premenopausal women with breast cancer $(\mathrm{RR}=1.10 ; 95 \%$ CI [1.07, 1.14], $\mathrm{p}<0.00001)$, compared to the control group (Figure 3).

\section{Ten years DFS of patients}

Ten clinical trials evaluated the 10 years DFS of pati- ents. Heterogeneity was considered absent at the I2 statistic ( $\mathrm{I} 2=41 \%, \mathrm{p}=0.15$ ). A fixed effects model was performed on outcome measurements. The results show that goserelin combined with chemotherapy could significantly increase the efficacy of 5 years DFS in premenopausal women with breast cancer $(\mathrm{RR}=$ $1.10 ; 95 \%$ CI $[1.07,1.14], \mathrm{p}<0.00001)$, compared to the control group (Figure 4).

\section{Five years PFS of patients}

Ten clinical trials evaluated the 5 years PFS of patients. Heterogeneity was considered absent at the I2 statistic (I2 $=0 \%, p=1.00)$. A fixed effects model was performed on outcome measurements. The results show that goserelin combined with chemotherapy could significantly increase the efficacy of 5 years PFS in premenopausal women with breast cancer $(\mathrm{RR}=1.11 ; 95 \%$ CI [1.08, 1.14], $\mathrm{p}<0.00001)$, compared to the control group 


\begin{tabular}{|c|c|c|c|c|c|c|c|c|c|}
\hline \multirow[b]{2}{*}{ Study or Subgroup } & \multicolumn{2}{|c|}{ Experiment group } & \multicolumn{2}{|c|}{ Control group } & \multirow[b]{2}{*}{ Weight } & Risk Ratio & \multicolumn{2}{|c|}{ Risk Ratio } & \\
\hline & Events & Total & Events & Total & & M.H, Fixed, 95\% Cl & \multicolumn{3}{|c|}{ M.H, Fixed, 95\% Cl } \\
\hline Allan Hackshaw 2009 & 422 & 469 & 405 & 476 & $9.0 \%$ & $1.06[1.01,1.11]$ & \multicolumn{3}{|c|}{-} \\
\hline Gunter von Minckwitz 2006 & 373 & 393 & 329 & 378 & $7.5 \%$ & $1.09[1.04,1.14]$ & \multicolumn{2}{|r|}{$\rightarrow$} & \\
\hline M. Baum 2006 & 1093 & 1354 & 1038 & 1356 & $23.1 \%$ & $1.05[1.01,1.10]$ & \multicolumn{2}{|r|}{$\rightarrow$} & \\
\hline M. Kaufmann 2003 & 617 & 735 & 571 & 758 & $12.5 \%$ & $1.11[1.06,1.17]$ & \multicolumn{2}{|r|}{$\rightarrow$} & \\
\hline Manfred Kaufmann 2007 & 340 & 384 & 332 & 392 & $7.3 \%$ & $1.05[0.99,1.10]$ & \multicolumn{2}{|r|}{-} & \\
\hline Monica C. Gertsch 2003 & 339 & 357 & 324 & 360 & $7.2 \%$ & $1.06[1.01,1.10]$ & \multicolumn{2}{|r|}{$\rightarrow$} & \\
\hline Nancy E. Davidson 2005 & 437 & 502 & 420 & 494 & $9.4 \%$ & $1.02[0.97,1.08]$ & \multirow{2}{*}{\multicolumn{2}{|c|}{$\rightarrow$}} & \\
\hline P. Karlsson 2011 & 332 & 354 & 311 & 389 & $6.9 \%$ & $1.08[1.03,1.14]$ & & & \\
\hline Tsui Fen Cheng 2012 & 137 & 152 & 334 & 412 & $4.0 \%$ & $1.11[1.04,1.19]$ & \multicolumn{2}{|r|}{$\longrightarrow$} & \\
\hline W. Jonat 2002 & 616 & 733 & 589 & 753 & $13.0 \%$ & $1.07[1.02,1.13]$ & \multicolumn{2}{|r|}{$\rightarrow$} & \\
\hline Total $(95 \% \mathrm{Cl})$ & & 5433 & & 5738 & $100.0 \%$ & $1.07[1.05,1.09]$ & \multicolumn{2}{|r|}{1} & \\
\hline Total events & \multicolumn{2}{|l|}{4706} & 4653 & & & & & & \\
\hline \multicolumn{3}{|c|}{ Heterogeneity: $\mathrm{Chi}^{2}=9.22, \mathrm{df}=9(\mathrm{p}=0.42) ; \mathrm{I}^{2}=2 \%$} & & & & & 1 & 1.5 & 2 \\
\hline \multicolumn{3}{|c|}{ Test for overall effect: $Z=7.99(p<0.00001)$} & & & & Control beneficia & \multicolumn{3}{|c|}{ Experimental beneficial } \\
\hline
\end{tabular}

Figure 2: Meta-analysis of 5 years OS

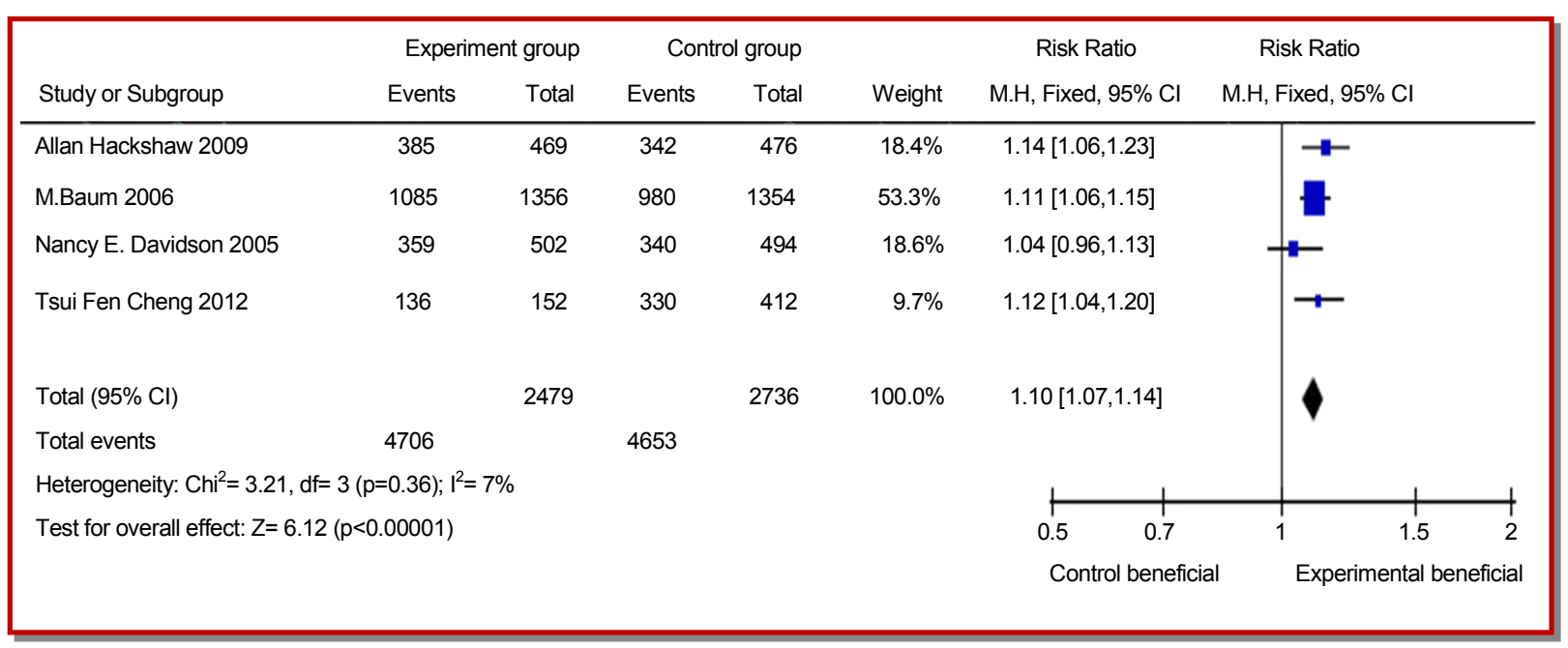

Figure 3: Meta-analysis of 10 years OS

(Figure 5).

\section{Emesis of patients}

Three clinical trials evaluated the emesis of patients. Heterogeneity was considered absent at the $\mathrm{I}^{2}$ statistic $\left(\mathrm{I}^{2}=18 \%, \mathrm{p}=0.30\right)$. A fixed effects model was performed on outcome measurements. The results show that goserelin combined with chemotherapy could significantly increase the incidence rate of emesis of premenopausal women with breast cancer ( $R R=2.01 ; 95 \%$ CI $[1.34,3.00]$, $\mathrm{p}=0.0007)$, compared to the control group (Figure 6).

\section{Arthralgia of patients}

Three clinical trials evaluated the arthralgia of patients. Heterogeneity was considered absent at the $\mathrm{I}^{2}$ statistic $\left(\mathrm{I}^{2}=0 \%, \mathrm{p}=0.46\right)$. A fixed effects model was performed on outcome measurements. The results show that goserelin combined with chemotherapy can significantly reduce arthralgia in premenopausal women with breast cancer $(R R=0.70 ; 95 \% C I[0.50,0.96], p=0.03)$, compared to the control group (Figure 7).

\section{Publication bias}

The shape of the funnel plot for the homozygote comparison appeared to some asymmetry and no obvious bias in this meta-analysis, suggesting the possibility of publication bias (Figure 8). Publication bias was assessed by Begg's test and Egger's test (Table II). No publication biases were observed except when comparing the efficiency of combination group and chemotherapy group on 5 years DFS (p-value [Begg's]: 0.023, p-value [Eegg's]: 0.003). It showed a potential publication bias might caused by a language bias, inflated estimates by a flawed methodological design in smaller studies, and/or a lack of publication of small trials with opposite results. 


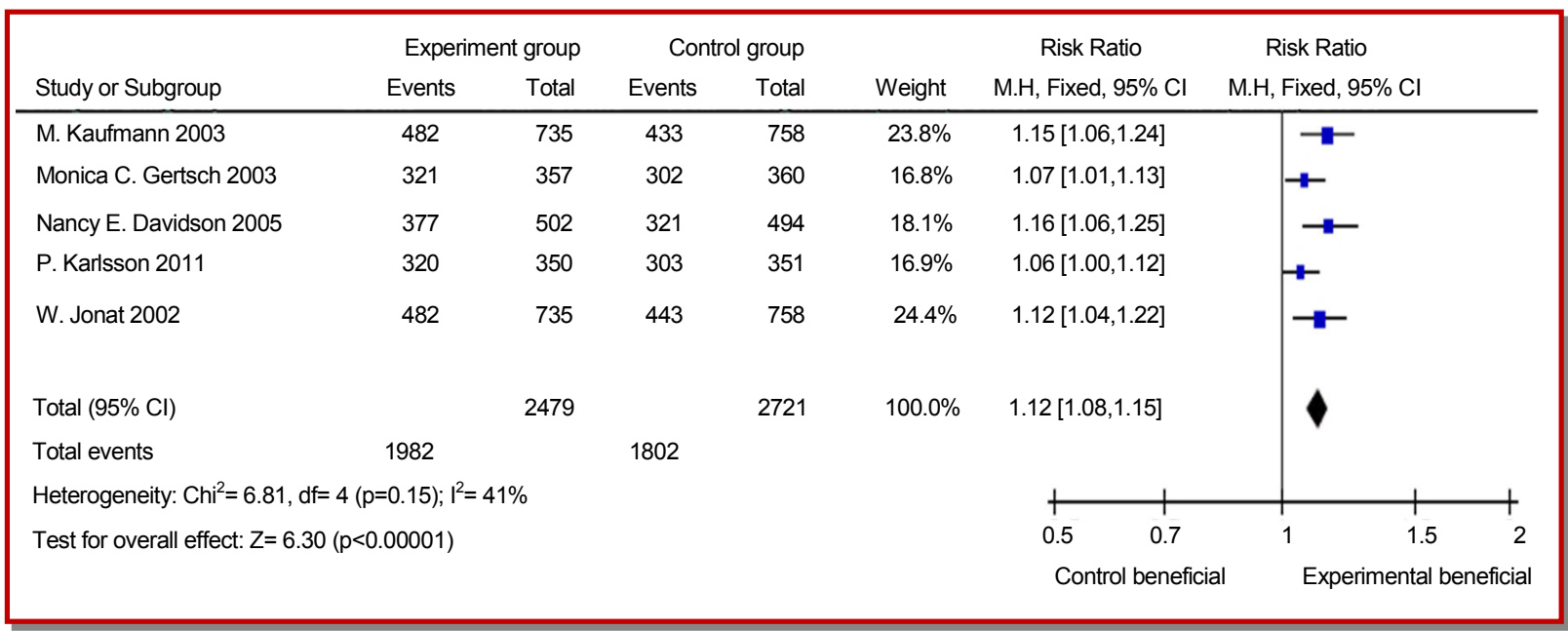

Figure 4: Meta-analysis of 5 years DFS

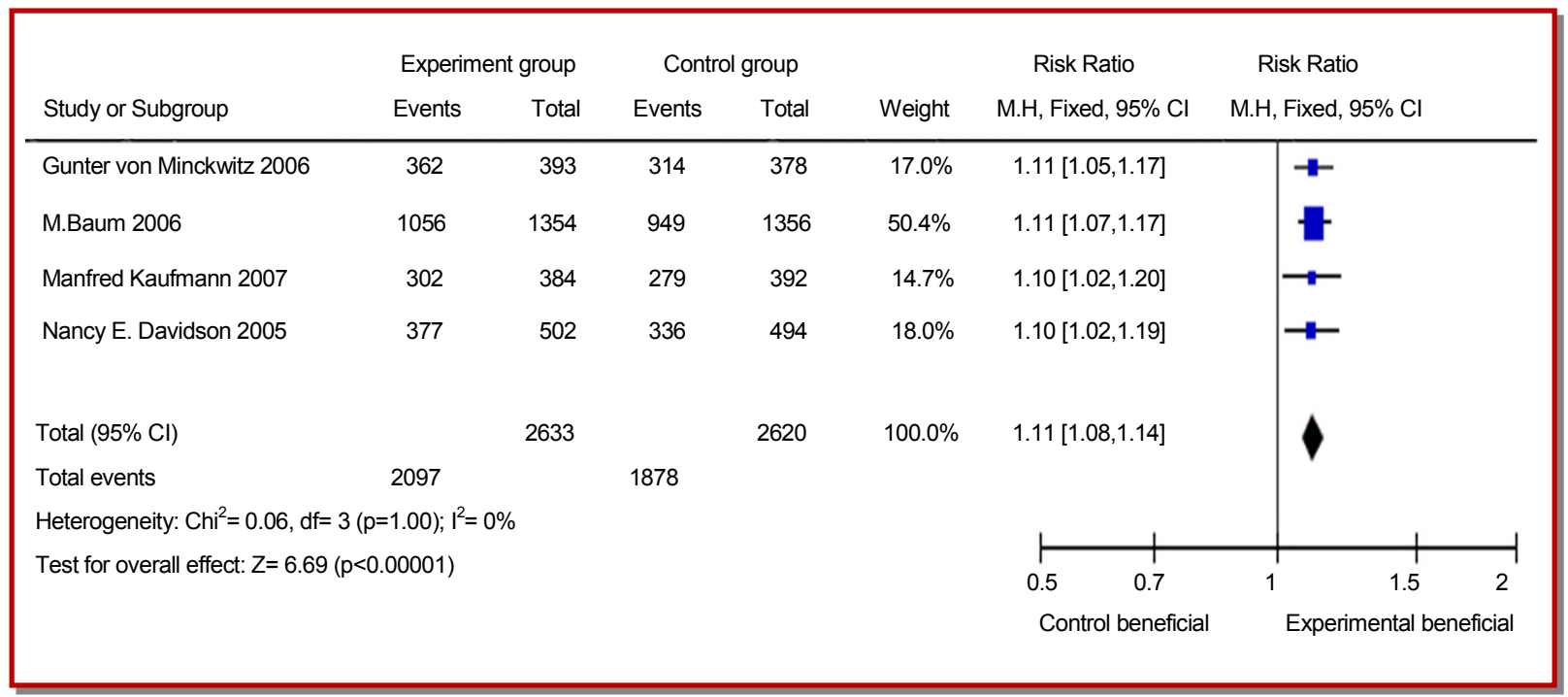

Figure 5: Meta-analysis of 5 years PFS

\section{Table II}

\section{Meta-analysis on efficacy and safety of GOS combined with chemotherapy}

\begin{tabular}{|c|c|c|c|c|c|c|c|c|c|}
\hline \multirow[t]{2}{*}{ Study } & \multirow[t]{2}{*}{ Indicator } & \multirow[t]{2}{*}{ Numberof trials } & \multicolumn{3}{|c|}{ Combined effect size } & \multicolumn{2}{|c|}{ Heterogeneity } & \multicolumn{2}{|c|}{ Publication bias } \\
\hline & & & RR & $95 \% \mathrm{CI}$ & Mode & $\mathrm{I}^{2}(\%)$ & p-Value & $\begin{array}{l}\text { p-Value } \\
\text { (Begg's) }\end{array}$ & $\begin{array}{l}\mathrm{p} \text {-Value } \\
\text { (Eegg's) }\end{array}$ \\
\hline \multirow{3}{*}{ Test of efficiency } & 5 years OS & 10 & 1.07 & $1.05-1.09$ & $\mathrm{~F}$ & 2 & 0.42 & 0.421 & 0.323 \\
\hline & 10 years OS & 4 & 1.10 & $1.07-1.14$ & $\mathrm{~F}$ & 7 & 0.36 & 0.500 & 0.233 \\
\hline & 5 years DFS & 5 & 1.10 & $1.07-1.14$ & $\mathrm{~F}$ & 41 & 0.15 & 0.023 & 0.003 \\
\hline \multirow{3}{*}{ Test of safety } & 5 years PFS & 4 & 1.11 & $1.08-1.14$ & F & 0 & 1.00 & 0.308 & 0.062 \\
\hline & emesis & 3 & 2.01 & $1.34-3.00$ & F & 18 & 0.30 & 0.602 & 0.410 \\
\hline & arthralgia & 4 & 0.70 & $0.50-0.96$ & $\mathrm{~F}$ & 0 & 0.46 & 0.734 & 0.225 \\
\hline
\end{tabular}




\begin{tabular}{|c|c|c|c|c|c|c|c|c|}
\hline \multirow[b]{2}{*}{ Study or Subgroup } & \multicolumn{2}{|c|}{ Experiment group } & \multicolumn{2}{|c|}{ Control group } & \multirow[b]{2}{*}{ Weight } & Risk Ratio & \multirow{2}{*}{$\begin{array}{l}\text { Risk Ratio } \\
\text { M.H, Fixed, 95\% Cl }\end{array}$} & \\
\hline & Events & Total & Events & Total & & M.H, Fixed, $95 \% \mathrm{Cl}$ & & \\
\hline M. Baum 2006 & 9 & 134 & 2 & 137 & $6.0 \%$ & $4.60[1.01,20.90]$ & $\longrightarrow$ & \\
\hline Manfred Kaufmann 2007 & 1 & 384 & 2 & 392 & $6.0 \%$ & $0.51[0.05,5.61] \longrightarrow$ & & \\
\hline Nancy E. Davidson 2005 & 57 & 50 & 29 & 494 & $88.1 \%$ & $1.93[1.26,2.97]$ & & \\
\hline Total $(95 \% \mathrm{Cl})$ & & 1020 & & 1023 & $100.0 \%$ & $2.01[1.34,3.00]$ & 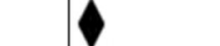 & \\
\hline Total events & 67 & & 33 & & & & & \\
\hline \multicolumn{9}{|c|}{ Heterogeneity: $\mathrm{Chi}^{2}=2.44, \mathrm{df}=2(\mathrm{p}=0.30) ; \mathrm{I}^{2}=18 \%$} \\
\hline \multirow{2}{*}{\multicolumn{2}{|c|}{ Test for overall effect: $Z=3.40(p<0.00001)$}} & & & & & 0.001 & 10 & 1000 \\
\hline & & & & & & Control beneficial & Experimental & neficial \\
\hline
\end{tabular}

Figure 6: Meta-analysis of emesis

\begin{tabular}{|c|c|c|c|c|c|c|c|c|c|}
\hline \multirow[b]{2}{*}{ Study or Subgroup } & \multicolumn{2}{|c|}{ Experiment group } & \multicolumn{2}{|c|}{ Control group } & \multirow[b]{2}{*}{ Weight } & \multirow{2}{*}{$\begin{array}{c}\text { Risk Ratio } \\
\text { M.H, Fixed, } 95 \% \mathrm{Cl}\end{array}$} & \multirow{2}{*}{\multicolumn{2}{|c|}{$\begin{array}{c}\text { Risk Ratio } \\
\text { M.H, Fixed, } 95 \% \mathrm{Cl}\end{array}$}} & \\
\hline & Events & Total & Events & Total & & & & & \\
\hline M. Baum 2006 & 2 & 134 & 6 & 137 & $7.0 \%$ & $0.34[0.07,1.66]$ & — & + & \\
\hline Monica C. Gertsch 2003 & 7 & 357 & 11 & 360 & $13.0 \%$ & $0.64[0.25,1.64]$ & & & \\
\hline Nancy E. Davidson 2005 & 41 & 502 & 48 & 494 & $57.4 \%$ & $0.84[0.56,1.25]$ & & & \\
\hline W. Jonat 2002 & 9 & 802 & 19 & 802 & $22.5 \%$ & $0.47[0.22,1.04]$ & & 7 & \\
\hline Total $(95 \% \mathrm{Cl})$ & & 1795 & & 1793 & $100.0 \%$ & $0.70[0.50,0.96]$ & & 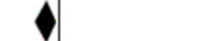 & \\
\hline Total events & 59 & & 84 & & & & & & \\
\hline \multicolumn{6}{|c|}{ Heterogeneity: $\mathrm{Chi}^{2}=2.59, \mathrm{df}=3(\mathrm{p}=0.46) ; \mathrm{I}^{2}=0 \%$} & & & + & -1 \\
\hline \multirow{2}{*}{\multicolumn{2}{|c|}{ Test for overall effect: $Z=2.19(p<0.03)$}} & & & & & 0.001 & 0.1 & 10 & 1000 \\
\hline & & & & & & Control benefi & ficial & Experimenta & neficial \\
\hline
\end{tabular}

Figure 7: Meta-analysis of arthralgia

\section{Discussion}

As a hormone-dependent malignant tumor, breast cancer is a serious threat to women's health, of which the incidence rate is increasing (Nishimura et al., 2013). Breast tumor cells are influenced by hormone levels in the processes of growth and proliferation. It is found from researching that the incidence, the development and prognosis as well as the therapy effect of breast cancer are closely correlated with the expressions of the estrogen receptor and progesterone receptor.

Goserelin is a synthetic analogue of a naturally occurring luteinizing-hormone releasing hormone, and the long-term use can inhibit the luteinizing-hormone secretion of pituitary, thus causing a decline in male serum testosterone and female serum estradiol (Nishimura et al., 2013; Zhou et al., 2013). After 21 days from the initial medical treatment for female patients, serum estradiol concentration is inhibited, which maintains in postmenopausal level during each 28-day treatment thereafter. This inhibition is related to hormone-dependent breast cancer and endometriosis. There may be some symptoms such as flushing, sweating and loss of libido appearing in female patients, and generally it is not necessary to stop the medicine. Headaches and mood changes such as depression may also be seen, as well as vaginal dryness and changes in breast volume. Patients with breast cancer will suffer from the intensified symptoms in the early stage of having the medicine. An extremely small number of patients suffering from endometriosis enter menopause, and their menses will not be recovered after stopping the analogue of luteinizing-hormone releasing hormone (Mills et al., 2005).

From the study of this paper, it is found that goserelin combined with chemotherapy can improve the survival rate and decrease the incidence of arthralgia in postmenopausal breast cancer patients during the chemotherapy process. However, they can increase the occurrence of vomiting in postmenopausal breast cancer patients during the chemotherapy process, which is notable. These characteristics indicate researching direc- 


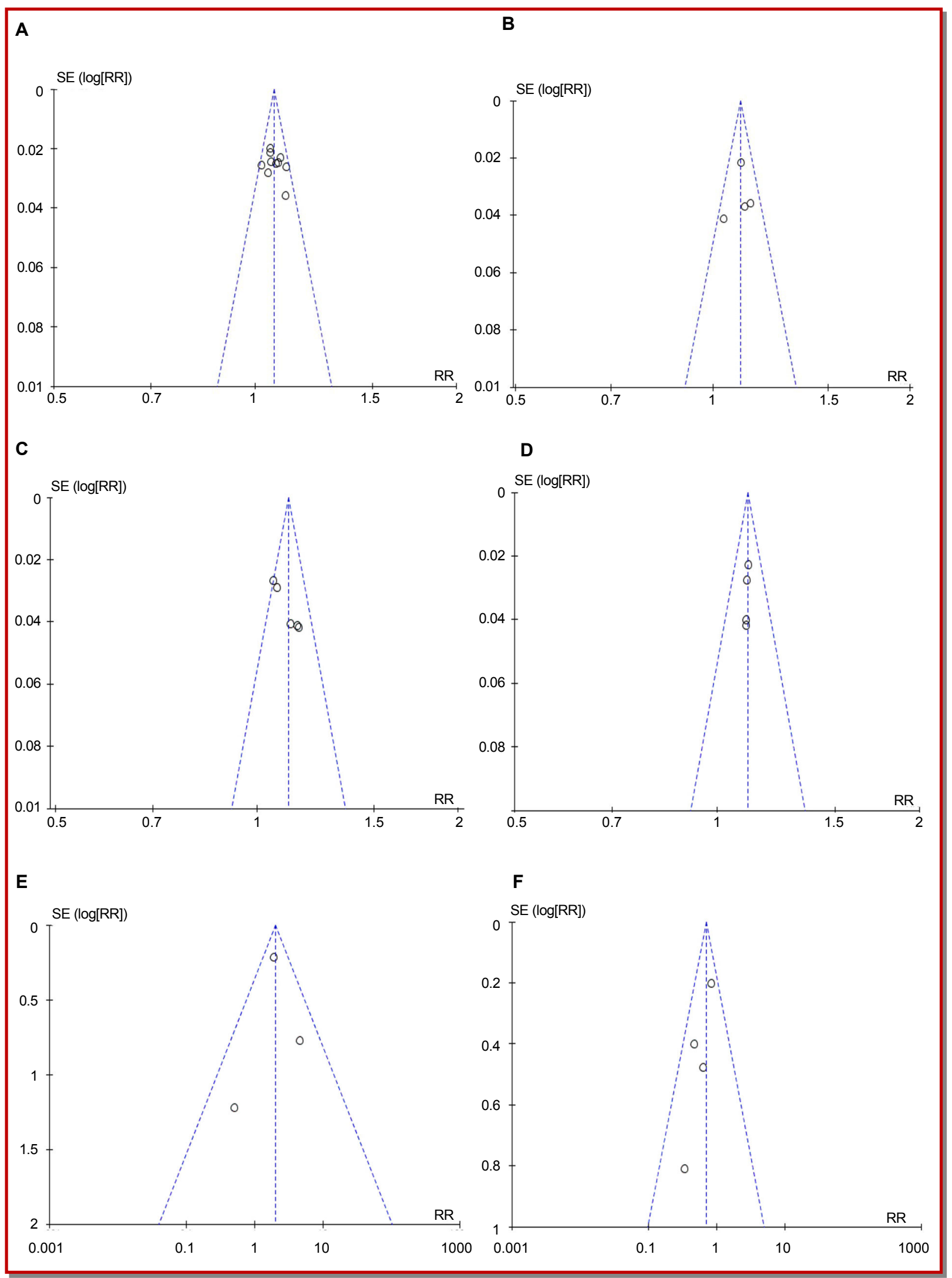

Figure 8: Funnel plot of the studies comparing the efficiency. 5 years OS (A); 10 years OS (B); 5 years DFS (C); 5 years PFS (D); emesis (E); arthralgia (F) 
tion of goserelin combined with chemotherapy. But the symptoms may also be caused by the small sample size of this experiment, which is expected to be improved in the future.

The number of people studied as research objects is up to 2710 , and 465 at least; the study of which the sample size is too small may achieve the low accuracy of the test result, with the increased incidence rated of type II error, which is the inadequacy of this study (Moher et al., 2012). The average number of cases in experimental group is 54.3, while the average number of cases in control group is 573.8; the research objects in both groups are premenopausal breast cancer patients. And the numbers of objects in all studies are more than 100 , but two of them include the estimation of sample size. All RCTs included in this study describe specific stochastic approach. In three papers, hide methods of allocation have been reported, and there are seven papers in the literature have mentioned double-blind method, but have not adopted intention principles of treatment for data analysis. Hide allocation plan is equally important for the prevention from bias and randomization, and the allocation without hide can make the effect of the intervention exaggerated by 30$41 \%$ on average (Karlsson et al., 2011), which is a deficiency in this study. All the previous studies made a comparison of the baseline information on pathological grading and staging, treatment programs of the patients, of which the results showed comparability of baseline between experimental and control groups ( $\mathrm{p}>$ 0.05).

Very few papers are included in the study of this paper as literature. The monitoring intervention for adverse medicine reactions is very significant for the evaluation of the medicine efficacy and clinical medicine administration. However, few reports included in the study mentioned adverse reactions, indicating that the researchers did not pay sufficient attention to the observation and the report of adverse reactions, which is not helpful to the promotion and application of goserelin combined with chemotherapy treatment of premenopausal breast cancer.

\section{Conclusion}

In this study, for the process of goserelin combined with chemotherapy treatment of pre-menopausal breast cancer patients, full consideration should be given to the rudimentary status of the disease, since disease category, disease condition, assessment of nutritional status, physical condition, comorbidities, complications, and the degree of primary disease are all the main factors affecting the treatment. Comparability of studied baseline should be guaranteed, research design should be standardized further, and RCTs should be reported particularly in accordance with CONSORT standard (Schulz et al., 1995). Future research will be more concerned about the impact of the goserelin in combination with chemotherapy on premenopausal breast cancer, in order to improve the quality of research. It is recommended that future studies avoid low-level repetition, and comply with the standards of randomized double-blind test with multi-center and large sample to design experiments; the reports about clinical trials of negative results should be emphasized; it is expected to obtain more reliable conclusions drawn for clinical applications.

\section{References}

Ai Z, Ning X, Shou T, Tang W, Luo Y, Zhang J. Association of interleukin-6 promoter polymorphism with knee osteoarthritis: A meta-analysis. Chin Med J (Engl). 2014; 127: 249296.

Baum M, Hackshaw A, Houghton J, Rutqvist, Fornander T, Nordenskjold B, Nicolucci A, Sainsbury R. Adjuvant goserelin in pre-menopausal patients with early breast cancer: Results from the ZIPP study. Eur J Cancer. 2006; 42: 895-904.

Castiglione-Gertsch M, O'Neill A, Price KN, Goldhirsch A, Coates AS, Colleoni M, Nasi ML, Bonetti M, Gelber RD. Adjuvant chemotherapy followed by goserelin versus either modality alone for premenopausal lymph node-negative breast cancer: a randomized trial. J Natl Cancer Inst. 2003; 95: 1833-46.

Chai Q, Liu J. Early stage effect of ischemic preconditioning for patients undergoing on-pump coronary artery bypass grafts surgery: Systematic review and meta-analysis. Pak J Med Sci. 2014; 30: 642-48.

Cheng TF, Wang JD, Uen WC. Cost-utility analysis of adjuvant goserelin (Zoladex) and adjuvant chemotherapy in premenopausal women with breast cancer. BMC Cancer. 2012; 12: 33.

Davidson NE, O'Neill AM, Vukov AM, Osborne CK, Martino $\mathrm{S}$, White DR, Abeloff MD. Chemoendocrine therapy for premenopausal women with axillary lymph node-positive, steroid hormone receptor-positive breast cancer: Results from INT 0101 (E5188). J Clin Oncol. 2005; 23: 5973-82.

DeSantis C, Ma J, Bryan L, Jemal A. Breast cancer statistics, 2013. CA Cancer J Clin. 2014; 64: 52-62.

Gerber B, von Minckwitz G, Stehle H, Reimer T, Felberbaum R, Maass N, Fischer D, Sommer HL, Conrad B, Ortmann O, Fehm T, Rezai M, Mehta K, Loibl S. Effect of luteinizing hormone-releasing hormone agonist on ovarian function after modern adjuvant breast cancer chemotherapy: The GBG 37 ZORO study. J Clin Oncol. 2011; 29: 2334-41.

Gielen SC, Kuhne LC, Ewing PC, Blok LJ, Burger CW. Tamoxifen treatment for breast cancer enforces a distinct geneexpression profile on the human endometrium: An exploratory study. Endocr Relat Cancer. 2005; 12: 1037-49.

Hackshaw A, Baum M, Fornander T, Nordenskjold B, Nicolucci A, Monson K, Forsyth S, Reczko K, Johansson U, Fohlin H, Valentini M, Sainsbury R. Long-term effectiveness of adjuvant goserelin in premenopausal women with early breast cancer. J Natl Cancer Inst. 2009; 101: 341-49. 
Higgins JP, Thompson SG. Quantifying heterogeneity in a meta-analysis. Stat Med. 2002; 21: 1539-58.

Hong-Li C, Xiao-Chun W, Jiang-Bin W, Jing-Bo Z, Yao W. Quality of life in patients with breast cancer and their rehabilitation needs. Pak J Med Sci. 2014; 30: 126-30.

Karlsson P, Sun Z, Braun D, Price KN, Castiglione-Gertsch M, Rabaglio M, Gelber RD, Crivellari D, Collins J, Murray E, Zaman K, Colleoni M, Gusterson BA, Viale G, Regan MM, Coates AS, Goldhirsch A. Long-term results of International Breast Cancer Study Group Trial VIII: Adjuvant chemotherapy plus goserelin compared with either therapy alone for premenopausal patients with node-negative breast cancer. Ann Oncol. 2011; 22: 2216-26.

Kaufmann M, Graf E, Jonat W, Eiermann W, Vescia S, Geberth M, Conrad B, Gademann G, Albert US, Loibl S, von Minckwitz G, Schumacher M. A randomised trial of goserelin versus control after adjuvant, risk-adapted chemotherapy in premenopausal patients with primary breast cancer - GABG-IV B-93. Eur J Cancer. 2007; 43: 2351-58.

Kaufmann M, Jonat W, Blamey R, Cuzick J, Namer M, Fogelman I, de Haes JC, Schumacher M, Sauerbrei W. Survival analyses from the ZEBRA study. goserelin (Zoladex) versus $\mathrm{CMF}$ in premenopausal women with node-positive breast cancer. Eur J Cancer. 2003; 39: 1711-17.

Liu X, Qu H, Cao W, Wang Y, Ma Z, Li F, Wang H. Efficacy of combined therapy of goserelin and letrozole on very young women with advanced breast cancer as first-line endocrine therapy. Endocr J. 2013; 60: 819-28.

Lyu J, Yang H, Lang J, Tan X. Tumor necrosis factor gene polymorphisms and endometriosis in Asians: A systematic review and meta-analysis. Chin Med J (Engl). 2014; 127: 1761 $-67$.

Mills EJ, Wu P, Gagnier J, Devereaux PJ. The quality of randomized trial reporting in leading medical journals since the revised CONSORT statement. Contemp Clin Trials. 2005; 26: 480-87.

Moher D, Hopewell S, Schulz KF, Montori V, Gotzsche PC,
Devereaux PJ, Elbourne D, Egger M, Altman DG. CONSORT 2010 explanation and elaboration: Updated guidelines for reporting parallel group randomised trials. Int J Surg. 2012; 10: $28-55$.

Nishimura R, Anan K, Yamamoto Y, Higaki K, Tanaka M, Shibuta K, Sagara Y, Ohno S, Tsuyuki S, Mase T, Teramukai $\mathrm{S}$. Efficacy of goserelin plus anastrozole in premenopausal women with advanced or recurrent breast cancer refractory to an LH-RH analogue with tamoxifen: Results of the JMTO BC08-01 phase II trial. Oncol Rep. 2013; 29: 1707-13.

Schulz KF, Chalmers I, Hayes RJ, Altman DG. Empirical evidence of bias. Dimensions of methodological quality associated with estimates of treatment effects in controlled trials. JAMA. 1995; 273: 408-12.

von Minckwitz G, Graf E, Geberth M, Eiermann W, Jonat W, Conrad B, Brunnert K, Gerber B, Vescia S, Wollert J, Kaufmann M. CMF versus goserelin as adjuvant therapy for node-negative, hormone-receptor-positive breast cancer in premenopausal patients: A randomised trial (GABG trial IVA-93). Eur J Cancer. 2006; 42: 1780-88.

Yang H, Zong X, Yu Y, Shao G, Zhang L, Qian C, Bian Y, Xu X, Sun W, Meng X, Ding X, Chen D, Zou D, Xie S, Zheng Y, Zhang J, He X, Sun C, Yu X, Ni J. Combined effects of goserelin and tamoxifen on estradiol level, breast density, and endometrial thickness in premenopausal and perimenopausal women with early-stage hormone receptor-positive breast cancer: A randomised controlled clinical trial. Br J Cancer. 2013; 109: 582-88.

Yang T, Yang X, Wang X, Wang Y, Song Z. The role of tomato products and lycopene in the prevention of gastric cancer: $\mathrm{A}$ meta-analysis of epidemiologic studies. Med Hypotheses. 2013; 80: 383-88.

Zhou J, Chen Y, Huang Y, Long J, Wan F, Zhang S. Serum follicle-stimulating hormone level is associated with human epidermal growth factor receptor type 2 and Ki67 expression in post-menopausal females with breast cancer. Oncol Lett. 2013; 6: 1128-32.

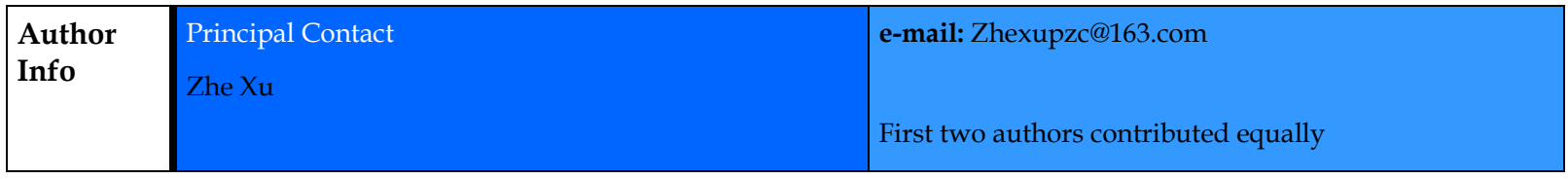




\section{Your feedback about this paper}

1. Number of times you have read this paper 0

2. Quality of paper
Excellent
Good
Moderate
Not good

3. Your comments 